\title{
Attentional Effects of Counterpredictive Gaze and Arrow Cues
}

\author{
Chris Kelland Friesen \\ North Dakota State University
}

\author{
Jelena Ristic and Alan Kingstone \\ University of British Columbia
}

\begin{abstract}
The authors used counterpredictive cues to examine reflexive and volitional orienting to eyes and arrows. Experiment 1 investigated the effects of eyes with a novel design that allowed for a comparison of gazed-at (cued) target locations and likely (predicted) target locations against baseline locations that were not cued and not predicted. Attention shifted reflexively to the cued location and volitionally to the predicted location, and these 2 forms of orienting overlapped in time. Experiment 2 discovered that another well-learned directional stimulus, an arrow, produced a different effect: Attention was shifted only volitionally to the predicted location. The authors suggest that because there is a neural architecture specialized for processing eyes, gaze-triggered attention is more strongly reflexive than orienting to arrows.
\end{abstract}

Behavioral studies with healthy adults have indicated that the tendency to move attention to where someone else is looking is so fundamental that people will attend automatically to a location gazed at by a face on a computer screen, even when gaze direction does not predict where a target item may appear (e.g., Driver et al., 1999; Friesen \& Kingstone, 1998; Langton \& Bruce, 1999). More recently, a study by Kingstone, Friesen, and Gazzaniga (2000) revealed that this effect is lateralized to the hemisphere specialized for processing face and gaze information. These findings, coupled with the observation that gaze direction can convey a broad range of important social signals, have led to the suggestion that orienting to gaze direction may represent a special form of attention (Kingstone, Smilek, Ristic, Friesen, \& Eastwood, 2003; Langton, Watt, \& Bruce, 2000). In the present study, we investigated this hypothesis by examining attentional orienting in response to directional stimuli that were either gaze cues or arrow cues.

In their original gaze study with adults, Friesen and Kingstone (1998) reported that when a schematic face was presented in the

Chris Kelland Friesen, Department of Psychology, North Dakota State University; Jelena Ristic and Alan Kingstone, Department of Psychology, University of British Columbia, Vancouver, British Columbia, Canada.

Some of the findings reported in this article were presented at the annual meeting of the Cognitive Neuroscience Society, April 2000, San Francisco. This research was supported by awards to Chris Kelland Friesen from the Natural Sciences and Engineering Research Council of Canada (NSERC), the Alberta Heritage Foundation for Medical Research, and the Killam Trusts; by awards to Jelena Ristic from NSERC and the Michael Smith Foundation for Health Research (MSFHR); and by NSERC Grant 12R80338, MSFHR Grant 12R52471, and Human Frontier Science Program Grant 12R40417 to Alan Kingstone. We thank Bruce Hood for his very helpful comments, and we acknowledge the many contributions of G. D. Millstone since we began work on this study.

Correspondence concerning this article should be addressed to Chris Kelland Friesen, Department of Psychology, College of Science and Mathematics, North Dakota State University, P.O. Box 5075, Fargo, ND 581055075, or to Alan Kingstone, Department of Psychology, University of British Columbia, 2136 West Mall, Vancouver, British Columbia V6T 1Z4, Canada. E-mail: chris.friesen@ndsu.nodak.edu or alan.kingstone@ubc.ca center of a computer screen, and the gaze direction of the face was known to be spatially nonpredictive, adults were nevertheless faster to detect, localize, and identify a target stimulus if it appeared at the location that the face was looking at rather than at a non-gazed-at location. An equally interesting result was that this facilitatory effect of gaze direction emerged soon after the schematic eyes were presented-at a cue-target stimulus onset asynchrony (SOA) of $105 \mathrm{~ms}$ - and then persisted across SOAs of 300 and $600 \mathrm{~ms}$ before disappearing by a 1,005-ms SOA. Both the rapid onset of the facilitation effect and the fact that it occurred in response to a nonpredictive stimulus are hallmarks of reflexive attentional orienting (Cheal \& Lyon, 1991; Jonides, 1981; Müller \& Rabbitt, 1989). This suggested to Friesen and Kingstone that they were measuring a reflexive attentional phenomenon.

However, Friesen and Kingstone (1998) also noted that orienting to gaze direction did not exhibit all of the characteristics normally associated with reflexive shifts of attention. For instance, in their study, the attentional shift to a peripheral location was triggered by a spatially nonpredictive stimulus (the eyes) presented at central fixation. In contrast, reflexive orienting is normally produced by presenting a spatially nonpredictive transient event, such as the brightening of a box, at a peripheral location where a target might appear (e.g., Posner \& Cohen, 1984; Posner, Cohen, $\&$ Rafal, 1982). Another difference was that Friesen and Kingstone found that orienting to gaze direction persisted well beyond a cue-target SOA of $500 \mathrm{~ms}$. The reflexive orienting effect produced by nonpredictive peripheral cues disappears when the cue-target SOA exceeds approximately $300 \mathrm{~ms}$ (Klein, Kingstone, \& Pontefract, 1992). A final difference was that when the facilitatory effect of gaze direction disappeared, it was never replaced by the inhibition of return (IOR) effect, an increase in response time (RT) for targets appearing at the cued location. This contrasts with spatially nonpredictive peripheral cuing, in which the short-lived early facilitation effect at the cued location is typically replaced by an IOR effect at longer SOAs (Posner \& Cohen, 1984; Posner, Rafal, Choate, \& Vaughan, 1985; for a review, see Klein, 2000). Considered together, these differences suggested to Friesen and King- 
stone that attention to gaze direction might represent a new and different type of reflexive orienting.

Similar findings and conclusions were put forward by Langton and Bruce (1999) and Driver et al. (1999). In addition, each of these two studies examined volitional orienting to gaze direction by testing performance when gaze direction predicted where a target stimulus was likely to appear. Langton and Bruce (1999, Experiment 3) examined volitional orienting by presenting an image of a real face in the center of the computer screen. The face could be turned to the left, to the right, up, or down. Subjects were informed that the target stimulus would appear $75 \%$ of the time at the location that the head and eyes were directed toward (the cued location) and $25 \%$ of the time at one of the three uncued target locations. Results indicated that RT was facilitated for targets appearing at the cued location both when the cue-target SOA was short $(100 \mathrm{~ms})$ and when it was long (1,000 ms). Langton and Bruce suggested that the facilitation observed at the short SOA reflected a reflexive shift of attention to the gazed-at location (because this effect was also observed at the short SOA in their first two experiments with nonpredictive gaze) and that the facilitation effect observed at the long SOA reflected voluntary orienting to the gazed-at location (because the attentional effect had disappeared at this long SOA in their nonpredictive gaze experiments). This account is both reasonable and consistent with the data. However, because Langton and Bruce sampled performance at only two temporal extremes-a short, 100-ms SOA, at which gaze-triggered reflexive orienting is often observed, and a long, 1,000 -ms SOA, at which gaze-triggered reflexive orienting is often absent - their results do not indicate when voluntary orienting in response to the predictive cue emerged or, more specifically, whether this voluntary orienting effect replaced reflexive orienting.

Driver et al. (1999, Experiment 3) tested the reflexivity of orienting to gaze direction by making the gaze cue counterpredictive with respect to where a target was likely to appear. Subjects were presented with an image of a real face pointed straight ahead but with eyes gazing to the left or right. They were informed that when the eyes looked to the left, the target would appear on the right $80 \%$ of the time, and vice versa. RT performance was sampled at 100-, 300-, and 700-ms cue-target SOAs. No effects of gaze direction were observed at the shortest SOA of $100 \mathrm{~ms}$; however, at the 300-ms SOA, RT was shorter at the location that the eyes were directed toward (where the target was unlikely to appear), and at the 700-ms SOA, there was a nonsignificant trend for RT to be shorter at the location opposite to where the eyes were directed (where the target was likely to appear). In keeping with Langton and Bruce (1999), Driver et al. suggested that at the shorter, 300-ms SOA, attention was reflexively committed to where the eyes were looking; conversely, at the longer, 700-ms SOA, attention was voluntarily shifted to the location where the target was likely to appear (in this case, the opposite location). Although this is a plausible interpretation of the data, the fact remains that performance was never significantly faster at the non-gazed-at (but likely) target location, and therefore the evidence does not provide strong support for the view that the reflexive orienting observed at the $300-\mathrm{ms}$ SOA was replaced by volitional orienting at the $700-\mathrm{ms}$ SOA.

One alternative interpretation is that with counterpredictive gaze cues, the conflict between the attentional effects of gaze direction and the task requirement to shift attention in the opposite direction somehow diminishes or delays volitional orienting (which usually emerges at cue-target SOAs of about 500 ms or less; Danziger \& Kingstone, 1999; Müller \& Rabbitt, 1989). A second alternative is that both reflexive orienting to gaze direction and voluntary orienting to the likely target location were occurring at the 700-ms SOA. If this were the case, the overall result might be to facilitate RT performance both at the gazed-at location (because of reflexive orienting) and at the likely location (because of volitional orienting), thereby reducing or eliminating any significant differences between these two locations. Note that this alternative interpretation is reasonable because both Driver et al. (1999, Experiments 1 and 2) and Friesen and Kingstone (1998) demonstrated originally with nonpredictive gaze cues that reflexive orienting can be observed with cue-target SOAs as long as 600-700 ms.

Considered as a whole, the data from these studies do not provide a clear picture of reflexive and voluntary orienting in response to central gaze-direction cues. The results of Langton and Bruce (1999) indicate that subjects can orient attention both reflexively and volitionally in response to predictive gaze cues, but they do not reveal whether volitional orienting replaces or overlaps with reflexive orienting. Similarly, the findings of Driver et al. (1999) with counterpredictive gaze cues indicate that at a short SOA of $300 \mathrm{~ms}$, attention is oriented reflexively to the gazed-at location even when subjects have an incentive to shift their attention in the direction opposite to where the eyes are looking, suggesting that orienting to gaze direction may be strongly reflexive. And the trend toward an RT advantage for targets appearing at the predicted location at the $700-\mathrm{ms}$ SOA suggests that some volitional orienting might have been occurring at this longer SOA. However, for the reasons just discussed, it is equivocal whether the nonsignificant benefit for predicted (but not gazed-at) targets at the 700-ms SOA simply reflects weak or delayed volitional orienting or the fact that reflexive orienting and volitional orienting were co-occurring at this longer SOA.

In Experiment 1 of the present study, we used counterpredictive gaze cues in an improved design that allowed us to isolate reflexive orienting to a gazed-at location from voluntary orienting to a predicted location and to examine the time course of orienting to counterpredictive gaze direction across a wide range of SOAs. In Experiment 2, we examined the attentional effects of counterpredictive arrows in order to investigate whether the effects obtained with gaze cues in Experiment 1 would also be observed with common directional cue that was less biologically relevant.

\section{Experiment 1}

In Driver et al.'s (1999, Experiment 3) counterpredictive gaze experiment, subjects oriented reflexively to a gazed-at but unlikely target location at a short SOA of $300 \mathrm{~ms}$, but there was no clear evidence of a switch to volitional orienting to a likely target location at a longer SOA of $700 \mathrm{~ms}$. Two possible reasons for the absence of significant volitional orienting were advanced above. One possibility is that when gaze direction is counterpredictive, there is an inherent tension between reflexive and volitional orienting that delays or abolishes volitional orienting to the predicted location. A second possibility is that at an intermediate SOA, both forms of orienting might be operating independently, and when performance at the two locations is contrasted, there is no signif- 
icant difference because performance is facilitated by attention at both locations.

Experiment 1 tested these two ideas. To explore the first possibility, we extended the range of cue-target intervals to include long SOAs of 1,200 and 1,800 ms. We reasoned that this would provide ample opportunity for attention to be oriented volitionally to the predicted (but not gazed-at) location and that at these long SOAs, any reflexive tendency to orient toward the gazed-at location should no longer be present. We tested the second possibility by increasing the number of target locations from two to four. ${ }^{1}$ In this way, on any given trial, there would always be potential target locations that were neither gazed-at nor predicted. These locations would therefore provide a true baseline against which to assess the allocation of reflexive attention to the gazed-at location and volitional attention to the predicted location.

The gaze direction of a centrally presented schematic face served as the cue, and target onset could occur to the left of, to the right of, above, or below the face. To assess any transitions from reflexive orienting to volitional orienting, we measured performance at a short SOA (105 ms), at which reflexive orienting is typically observed; at an intermediate SOA (600 ms), at which both reflexive and volitional effects might occur; and at two long SOAs (1,200 and 1,800 ms), at which volitional attentional effects should predominate.

\section{Method}

Subjects. Twenty-four introductory psychology students (17 female and 7 male) reporting normal or corrected-to-normal vision participated in this experiment for course credit. All subjects were unaware of the purposes of the experiment. Testing was divided over two sessions of less than $1 \mathrm{hr}$ each, conducted on separate days.

Apparatus and stimuli. The experiment was controlled by VScope software (Version 1.2.7; Rensink, 1995) on a 6100 Power Macintosh computer. Stimuli were presented on a 14-in. (35.56-cm) Apple color monitor set to black and white and operating at a refresh rate of $66.7 \mathrm{~Hz}$. RT measures were based on keyboard responses.

The face display, illustrated in Figure 1, consisted of a black line drawing of a face presented on a white background. The round face outline subtended $6.8^{\circ}$ and contained two circles representing the eyes, a smaller circle in the center of the monitor representing the nose and serving as the fixation point, and a straight line representing the mouth. The eyes subtended $0.9^{\circ}$, and the center of each eye was located $1.0^{\circ}$ to the left or right of the central vertical axis and $0.8^{\circ}$ above the central horizontal axis. The

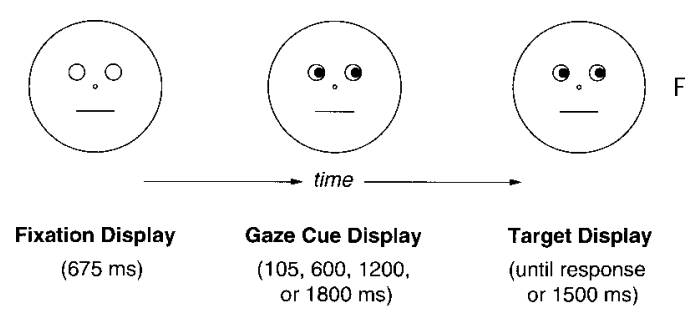

Figure 1. Illustration of the trial sequence in Experiment 1. Each trial began with the presentation of a face with blank eyes. After $675 \mathrm{~ms}$, pupils appeared in the eyes, looking left, right, up, down, or straight ahead (the gaze cue). Then, after $105,600,1,200$, or $1,800 \mathrm{~ms}$, the letter $F$ or $T$ (the target) appeared to the left or to the right of, above, or below the face. The target was likely to appear at the location opposite to the gazed-at location $75 \%$ of the time when the eyes looked left, right, up, or down. nose subtended $0.2^{\circ}$. The mouth was $2.2^{\circ}$ in length and was centered $1.3^{\circ}$ below the nose. Black filled-in circles appeared within the eyes and represented the pupils. The pupils subtended $0.5^{\circ}$. For the left and right gaze-direction cues, the pupils were centered vertically in the eyes and were just touching either the left or right of the eyes; for the up and down gaze directions, the pupils were centered horizontally in the eyes and were just touching either the top or the bottom of the eyes; and for the straightahead gaze, the pupils were centered both horizontally and vertically in the eyes. Note that when the pupils were presented, they simply appeared in a left, right, up, down, or straight gaze position, and thus there was no motion artifact.

The target stimulus demanding a detection response was a black capital letter $F$ or $T$ that measured $0.75^{\circ}$ wide and $1.35^{\circ}$ high and was presented to the left of, to the right of, above, or below the face. Target letters were centered on either the horizontal or vertical meridian, and the distance between central fixation (the nose) and the center of the target letter was $6.25^{\circ}$.

Design. Cue-target SOA $(105,600,1,200$, or 1,800 ms), gaze direction (left, right, up, down, or straight), and target identity ( $F$ or $T)$ were selected randomly and with equal probability. When gaze direction was left, right, up, or down, the target letter appeared at the location opposite to where the eyes were looking $75 \%$ of the time (e.g., if the eyes looked up, the target was most likely to appear below the face). If a target did not appear at the predicted location, target location was selected randomly and with equal probability from among the three remaining positions. When gaze direction was straight, a target was presented at one of the four locations (left, right, above, or below) randomly and with equal probability.

There were four trial types: (a) predicted trials, in which the target appeared at the predicted location (i.e., at the position opposite to the gazed-at location); (b) cued trials, in which the target appeared at the gazed-at location; (c) not predicted-not cued (NP-NC) trials, in which the target appeared at one of the two locations that were neither predicted nor gazed at; and (d) nondirectional straight-gaze trials, in which the eyes looked straight ahead and the target could appear at any of the four target locations. Figure 2 illustrates the probabilities of the possible target positions for predicted, cued, and NP-NC trial types.

Each of the two experimental sessions was composed of 20 practice trials followed by 12 blocks of 60 trials, for a total of 1,440 test trials per subject. Approximately $8 \%$ of the test trials were catch trials, randomly selected from the five gaze-direction cues.

Procedure. The sequence of events on a target trial is illustrated in Figure 1. All trials began with the presentation of a face with blank eyes. After $675 \mathrm{~ms}$, pupils appeared within the eyes, looking left, right, up, down, or straight ahead. Then, after 105, 600,1,200, or 1,800 ms, a target letter appeared to the left of, to the right of, above, or below the face. Both the gazing face and the target letter remained on the screen until a response was made or 1,500 ms had elapsed, whichever came first. RT was measured from the time of target onset. The intertrial interval was $675 \mathrm{~ms}$

\footnotetext{
${ }^{1}$ The addition of up and down target locations raises the possibility of introducing differential cuing effects, depending on whether orienting occurs on the horizontal axis or the vertical axis. In their study with real faces, Langton and Bruce (1999) found that although gaze-cuing effects occurred only on the horizontal axis with inverted faces (Experiment 4), the effects were equivalent on both axes with upright faces (Experiments 1-3). To rule out the possibility that there were axis effects in the present study, we conducted an analysis of variance (ANOVA) for each of the two experiments with the axis on which the target appeared (horizontal or vertical), SOA $(105,600,1,200$, or $1,800 \mathrm{~ms})$, and trial type (predicted, cued, or not predicted-not cued) as within-subject variables. There was no interaction between axis and trial type for either gaze cues $(F<1, p>.95)$ or arrow cues $(F<1.7, p>.20)$. Axis was therefore not considered a variable in the present study.
} 


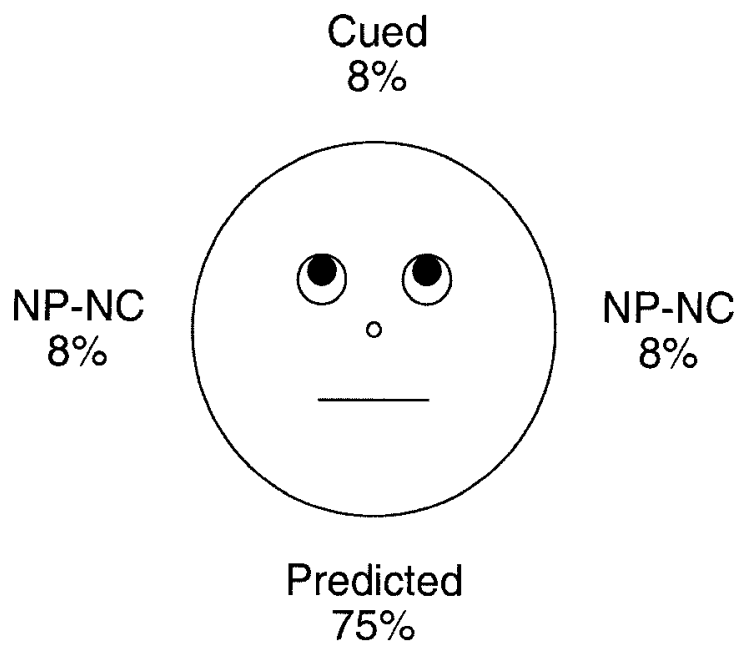

Figure 2. Illustration of the three trial types that were possible when gaze was directed at one of the four target locations in Experiment 1. Predicted $=$ target occurs at the predicted (not-cued) location. Cued $=$ target occurs at the cued (not-predicted) location. NP-NC $=$ target occurs at a location that is neither predicted nor cued. Percentages represent the probability (rounded to the nearest percentage point) of the target's appearance at each location.

Subjects were seated approximately $57 \mathrm{~cm}$ from the monitor, and the experimenter ensured that they were centered with respect to the monitor and keyboard. They were told that each trial would begin with a line drawing of a face with blank eyes; that pupils would appear in the eyes to create a face that was looking left, right, up, down, or straight ahead; and that after the appearance of the pupils, a capital letter (either $F$ or $T$ ) would typically appear to the left of, to the right of, above, or below the face. Subjects were instructed to press the spacebar with the index finger of their preferred hand as quickly as possible when a letter appeared on the screen. Also, they were told that occasionally there would be trials in which no target appeared, and on these trials they should not respond but just wait for the next trial to begin. The experimenter stressed that it was important to maintain fixation on the nose in the center of the face at all times.

It is important to note that the identity of the target was irrelevant to the response task-subjects were merely required to detect a target onset. Past research has demonstrated that when subjects are required to detect target onset, they usually will not move their eyes to the target before making a response (Posner, 1980). Indeed, it is difficult to get subjects to move their eyes before they make a manual detection response because it significantly slows their RT performance (Kingstone \& Pratt, 1999). Thus, we did not expect eye movements to be a confounding variable in our study. Moreover, Friesen and Kingstone (2003b) have confirmed that the standard attention effects produced by nonpredictive gaze direction do not depend on eye movements of the subjects. Nevertheless, we monitored the eye position of 7 subjects in this experiment, as well as in Experiment 2, to be certain that this was the case. As our results show, our expectations were confirmed: Subjects whose eye positions were monitored rarely executed eye movements before responding, and their performance was indistinguishable from the performance of those whose eyes were not monitored. Thus, the evidence indicates that eye movements rarely occurred in our study and are thus not an explanatory concern for our data.

Before the beginning of each session, subjects were told that $75 \%$ of the time that the eyes looked left, right, up, or down, the target letter would appear at the location opposite to where the eyes were looking and that when the eyes looked straight ahead, the target was equally likely to appear at any of the four possible target locations.

\section{Results}

Mean RTs, standard deviations, and error rates for Experiment 1 are presented in Table 1 . Anticipations (RTs $<100 \mathrm{~ms}$ ), timedout trials (no response), RTs longer than 1,000 ms, and incorrect responses (accidentally pressing a key other than the spacebar) were classified as errors and were excluded from analysis. Each type of error accounted for less than $0.5 \%$ of the target trials. The false-alarm rate on catch trials was $1.02 \%$. Because these rates were so low, the error and false-alarm data were not analyzed further.

An ANOVA was conducted with SOA $(105,600,1,200$, and $1,800 \mathrm{~ms}$ ) and trial type (predicted, cued, and NP-NC) as withinsubject variables. ${ }^{2}$ Mean RTs for predicted, cued, and NP-NC trials at each SOA are illustrated in Figure 3. The ANOVA revealed that there was a significant main effect for SOA, $F(3$, 23) $=66.66, M S E=404.09, p<.0001$, with RTs becoming shorter as SOA lengthened (a standard foreperiod effect; Friesen \& Kingstone, 1998; Mowrer, 1940). There was also a significant main effect for trial type, $F(2,23)=12.47, M S E=291.09, p<$ .0001 , with RTs being shortest on predicted trials, intermediate on cued trials, and longest on NP-NC trials. Finally, the SOA $\times$ Trial Type interaction was significant, $F(6,23)=2.66, M S E=170.55$, $p<.02$. Figure 3 suggests that this interaction reflects changes in the cued condition and the predicted condition relative to the NP-NC condition across SOAs. Specifically, it appears that for the cued condition, there was early facilitation that persisted steadily across the two early SOAs (105 and $600 \mathrm{~ms}$ ) and then disappeared at the 1,200-ms SOA. Conversely, for the predicted condition, facilitation emerged first at the $600-\mathrm{ms}$ SOA and persisted thereafter. Planned $t$ tests (Bonferroni corrected, two-tailed) confirmed these observations. For the cued condition, performance was significantly faster than for the NP-NC condition only at the 105- and 600-ms SOAs. Conversely, for the predicted condition, performance facilitation relative to NP-NC was absent at the 105-ms SOA and present at the 600-, 1,200-, and 1,800-ms SOAs.

Finally, the data for the 7 eye-monitored subjects revealed that the eye movement rate was low $(2.7 \%)$. RT performance for these 7 eye-monitored subjects was compared with that of the first 7 nonmonitored subjects we tested, using a two-within (SOA, trial type), one-between (eye-monitoring condition) ANOVA. The interactions involving monitoring condition and trial type fell far short of significance $\left(F_{\mathrm{s}}<1, p \mathrm{~s}>.50\right)$. Thus, we are certain that eye movements were not involved in producing the cuing effects and that the orienting we observed was covert in nature.

\footnotetext{
${ }^{2}$ Note that this ANOVA excluded straight-gaze trials and thus was not subject to any confounds inherent in comparing performance across different cue conditions (cf. Jonides \& Mack, 1984). In other words, for predicted, cued, and NP-NC trials, the cue conditions prior to target onset were identical, that is, averted gaze. In this way, similarities and differences between predicted, cued, and NP-NC can be attributed to attentional allocation without being compromised by factors such as changes in attentional set or response strategies between averted- and straight-gaze conditions. Performance on straight-gaze trials across both experiments is considered in detail in the General Discussion.
} 
Table 1

Mean Response Times (RTs; in Milliseconds), Standard Deviations, and Error Rates (\%E) for Experiment $1(N=24)$

\begin{tabular}{llll}
\hline $\begin{array}{l}\text { Cue-target SOA and } \\
\text { trial type }\end{array}$ & RT & $S D$ & $\% \mathrm{E}$ \\
\hline 105 ms & & & \\
Predicted & 390 & 55 & 0.94 \\
Cued & 385 & 51 & 0.93 \\
NP-NC & 397 & 58 & 0.85 \\
Straight-gaze & 396 & 56 & 0.99 \\
600 ms & & & \\
Predicted & 355 & 53 & 0.92 \\
Cued & 356 & 46 & 0.58 \\
NP-NC & 367 & 54 & 1.69 \\
Straight-gaze & 360 & 51 & 0.68 \\
1,200 ms & & & \\
Predicted & 338 & 50 & 0.48 \\
Cued & 354 & 55 & 0.56 \\
NP-NC & 356 & 49 & 0.93 \\
Straight-gaze & 347 & 46 & 0.69 \\
1,800 ms & & & \\
Predicted & 346 & 46 & 0.55 \\
Cued & 349 & 50 & 0.76 \\
NP-NC & 357 & 47 & 0.38 \\
Straight-gaze & 353 & 46 & 0.25 \\
\hline
\end{tabular}

Note. Error rates represent the percentage of test trials from each cell excluded as anticipations, keypress selection errors, timed-out trials, or trials with $\mathrm{RT}>1,000 \mathrm{~ms}$. SOA $=$ stimulus onset asynchrony; $\mathrm{NP}-\mathrm{NC}=$ not predicted-not cued.

\section{Discussion}

In the present counterpredictive gaze experiment, the use of four possible target locations made it possible to compare performance for targets appearing at a location that was gazed at but was unlikely to contain a target (cued trials) with RTs for targets appearing at a location that was not gazed at and yet was equally unlikely to contain a target (NP-NC trials). In a similar way, our design allowed us to compare RTs for targets appearing at a location that was not gazed at but was likely to contain a target (predicted trials) with RTs for targets appearing at a location that was not gazed at and was not likely to contain a target (NP-NC trials).

At the 105-ms SOA, responses to targets occurring at a gazed-at and unlikely location were significantly faster than responses to targets occurring at a non-gazed-at and unlikely location. This advantage for cued locations relative to the baseline locations that were neither predicted nor cued persisted at the 600-ms SOA and then disappeared by the 1,200-ms SOA. These findings are consistent with the reflexive effects observed with nonpredictive gaze cues (e.g., Driver et al., 1999; Friesen \& Kingstone, 1998; Langton \& Bruce, 1999), and they are also consistent with Driver et al.'s (1999, Experiment 3) finding that at a short SOA, gaze direction can produce a covert attention shift even when subjects have incentive based on cue predictiveness to shift attention to some other location.

We also observed clear evidence that subjects can shift attention volitionally to a predicted location when it is not the gazed-at location: Responses were reliably faster for predicted locations relative to locations that were neither predicted nor gazed at 600-, 1,200-, and 1,800-ms SOAs. Our observation of this effect at the 600-ms SOA indicates that counterpredictive gaze does not delay volitional orienting, a possibility that was suggested by the results of Driver et al.'s (1999, Experiment 3) counterpredictive gaze experiment. Rather, our observation suggests that in Driver et al.'s experiment, significant effects were not observed at the 700-ms SOA because both reflexive and volitional orienting were occurring at that cue-target interval, with reflexive attention being directed to the gazed-at location and volitional attention being directed to the predicted location. As a result, when these two locations were directly compared, there was no significant difference between them. Consistent with this notion, a direct comparison between predicted and cued trials in the present experiment revealed that the two were not statistically different at the $600-\mathrm{ms}$ SOA, $t(23)=0.38, p>.70$.

In sum, our data indicate that subjects will orient attention to a gazed-at location even though a target is unlikely to appear there and that they can also orient attention volitionally to a predicted, non-gazed-at location. Thus, Experiment 1 replicates Driver et al.'s (1999) finding that orienting to a gazed-at location is reflexive in the strong sense that it can occur even when subjects are trying to direct their attention to a different location, and it adds the new finding that subjects are indeed able to allocate attention volitionally to a location that is counter to the gazed-at location. In addition, it indicates why Driver et al. failed to observe a significant effect of volitional orienting with their counterpredictive gaze cues. That is, it appears that reflexive orienting to a gazed-at location and volitional orienting to a predicted location opposite to the gazed-at location overlap in time. Because in the counter predictive gaze experiment of Driver et al.'s study there were only two locations, facilitation effects at the gazed-at location and volitional orienting to the predicted location created the false impression that orienting was not occurring reliably at either location. The present experiment, which included baseline locations that were neither predicted nor cued, indicates that precisely the opposite is the case- - both volitional orienting to the predicted location and reflexive orienting to the gazed-at location can occur

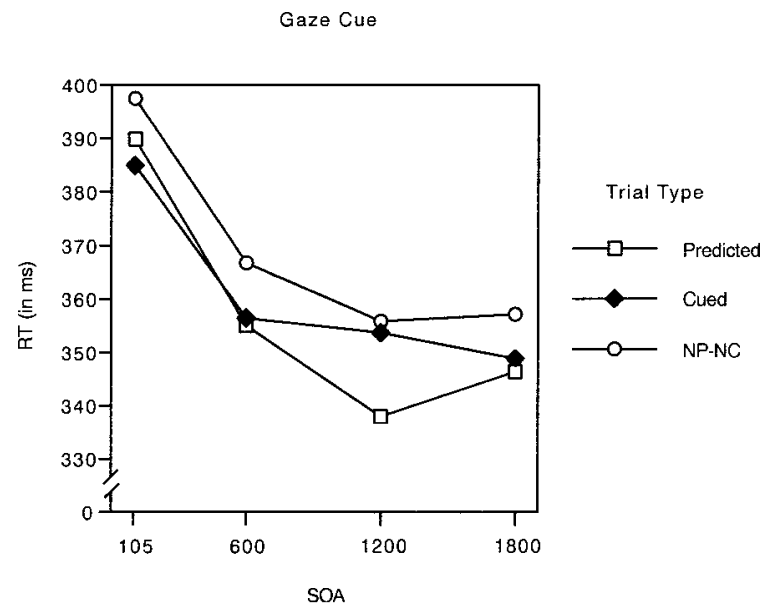

Figure 3. Mean response times (RTs) for counterpredictive gaze cues as a function of cue-target stimulus onset asynchrony (SOA) and trial type in Experiment 1. Predicted $=$ target occurs at the predicted (not-cued) location. Cued $=$ target occurs at the cued (not-predicted) location. NP-NC = target occurs at a location that is neither predicted nor cued. 
at an SOA (600 ms) that closely approximates the 700-ms SOA at which Driver et al.'s null finding was observed.

It is important to note that our finding that there was an RT advantage for both predicted and cued trials compared with NP-NC trials suggests that both forms of orienting might be operating concurrently. The design of the present experiment does not allow for a conclusive demonstration of simultaneous orienting to two different locations because, necessarily, on each trial only one location was probed. However, a closer examination of our data rules out the most plausible alternative explanations and favors our interpretation that our counterpredictive gaze cues produced concurrent reflexive and volitional orienting.

First, $t$ tests revealed that the reflexive cuing effect (NP-NC cued) at the 600-ms SOA was not statistically different from the reflexive effect at the 105-ms SOA, $t(23)=0.54, p>.58$, and that the volitional cuing effect (NP-NC - predicted) at the 600-ms SOA was not statistically different from the volitional effect at the 1,200 -ms SOA, $t(23)=1.51, p>.13$. This indicates that at the 600-ms SOA, RT benefits for the predicted target location were not replacing or occurring at the expense of RT benefits for the gazed-at target location.

Second, one could argue that our finding of facilitation on both predicted and cued trials at the $600-\mathrm{ms}$ SOA might reflect volitional orienting by roughly half of the subjects at the $600-\mathrm{ms}$ SOA and reflexive orienting by the other half of the subjects at the 600-ms SOA. The data at the 600-ms SOA, however, do not support this proposal: RTs for 15 subjects were shorter on both predicted and cued trials than on NP-NC trials, significantly more than would be expected by chance alone, $\chi^{2}(2, N=24), p<$ .0005 .

A third possible explanation for the RT advantage for both predicted and cued trials relative to NP-NC trials at the 600-ms SOA is that individual subjects were switching between the two types of orienting, that is, that they were shifting attention volitionally to the predicted location on some trials and shifting attention reflexively to the gazed-at location on other trials. If this were the case, then one would expect that the RT variance of the predicted and cued distributions would be greater than the RT variance of the NP-NC distribution. This is because on predicted and cued trials, sometimes the target would appear at an attended location and sometimes the target would appear at an unattended location. In contrast, on NP-NC trials, the variance would be lower because on every trial the target would appear at an unattended location. In other words, switching should result in greater variance because it entails the combined distribution of two component distributions with different means (attended and unattended target locations) compared with the baseline NP-NC condition, which has only one component distribution (unattended target locations). We tested this switching explanation by conducting an analysis of the within-subject standard deviations at the 600-ms SOA, and the results were clear-cut: Average standard deviations were not different between predicted trials $(81 \mathrm{~ms})$ and NP-NC trials $(78 \mathrm{~ms}), t(23)=0.74, p>.45$, and indeed, standard deviations were smaller on cued trials $(67 \mathrm{~ms})$ than on NP-NC trials, $t(23)=2.81, p<.01$. In agreement with this analysis, a visual inspection of individual subjects' RT distributions on predicted and cued trials at the $600-\mathrm{ms}$ SOA revealed no evidence of bimodality. In sum, our ability to rule out these alternative explanations favors the conclusion that reflexive orienting to the gazed-at location and volitional orienting to the predicted location can occur concurrently.

\section{Experiment 2}

The results of Experiment 1 indicate that orienting to gaze direction is reflexive in a strong sense. That is, target detection is facilitated at a gazed-at location despite the fact that gaze direction predicts that a target is likely to appear at a different, non-gazed-at location. And, most impressively, this facilitation occurs even when attention is being oriented volitionally to the predicted non-gazed-at location. Although it seems reasonable to speculate that the results of Experiment 1 are unique to gaze direction, this position remains untested. Indeed, recent evidence suggests that nonpredictive arrow cues can produce behavioral effects that look very similar to those produced by nonpredictive gaze cues (Ristic, Friesen, \& Kingstone, 2002; Tipples, 2002; for a discussion, see Kingstone, Smilek, Ristic, Friesen, \& Eastwood, 2003). The purpose of Experiment 2 was to examine whether similar effects to those observed in Experiment 1 would be observed with a counterpredictive central arrow cue.

Experiment 2 was identical in every way to Experiment 1, except that an arrow served as the counterpredictive directional cue. Two different arrows were used. One half of the subjects viewed a symmetrical arrow cue, with an arrow head at one end and a tail at the opposite end (e.g., $\longleftarrow$ ). The other half of the subjects viewed an asymmetrical arrow cue, with an arrow head at the leading end but with no tail (e.g., $\leftarrow$ ). This latter modification was made to examine whether gaze cues were strongly reflexive because they were perceptually weighted in the direction that they looked toward. For example, when the eyes are looking to the left, the pair of black pupils is not centered on the midline of the display but is instead centered on some point slightly to the left of the midline. If this is an important factor, then the symmetric and asymmetric arrows should produce different effects on reflexive attention (i.e., the asymmetrical arrow should produce stronger reflexive orienting).

\section{Method}

Subjects. Twenty-four introductory psychology students (20 female and 4 male) reporting normal or corrected-to-normal vision participated in the experiment for course credit. All subjects were unaware of the purposes of the experiment, and none had participated in Experiment 1. Testing was divided over two sessions of less than $1 \mathrm{hr}$ each, conducted on separate days. Eye monitoring was conducted as in Experiment 1.

Apparatus and stimuli. The apparatus was identical to that used in Experiment 1. Stimuli for Experiment 2 are illustrated in Figure 4. The fixation display consisted of a black line drawing of a cross centered within a circle. The circle subtended $6.8^{\circ}$ and was centered in the middle of the monitor. The cross within the circle was composed of a horizontal line and a vertical line, each of which was $2.1^{\circ}$ in length. The intersection of the two lines of the cross served as the fixation point. For half of the subjects, directional cues were provided by an arrow head and an arrow tail appearing at either end of one of the two lines of the cross (i.e., $1.2^{\circ}$ from central fixation, as measured from the intersection of the cross to the pointed end of the arrow head or tail); for the other half of the subjects, cues were provided by only an arrow head, appearing at one end of one of the two lines of the cross. The arrow heads (and tails) were composed of two lines that were $0.6^{\circ}$ in length and measured $0.8^{\circ}$ high $\times 0.5^{\circ}$ wide. A nondirectional cue (corresponding to the straight-gaze cue in Experiment 1) was 
A. Symmetrical Arrow Cue
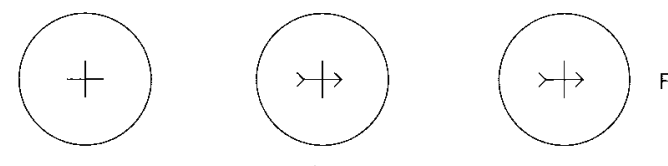

Fixation Display

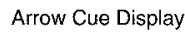

(675 ms)

$(105,600,1200$

or $1800 \mathrm{~ms}$ )

B. Asymmetrical Arrow Cue
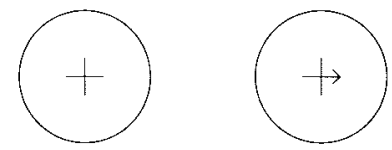

time
Target Display (until response or $1500 \mathrm{~ms})$

Figure 4. Illustration of the trial sequence in Experiment 2. Each trial began with a cross at central fixation. After $675 \mathrm{~ms}$, an arrow with a head and a tail (A) or an arrow with only a head (B) appeared on one of the two lines of the cross, creating an arrow pointing left, right, up, or down. On nondirectional cross trials, small perpendicular lines appeared at the ends of the lines of the cross. Then, after 105, 600,1,200, or 1,800 ms, a target letter $(F$ or $T$ ) appeared to the left of, to the right of, above, or below the cross. Trial types and probabilities were the same as those for counterpredictive gaze direction cues (see Figure 2).

provided by small lines appearing at the ends of both lines of the cross, such that each arm of the cross ended in a perpendicular line measuring $0.6^{\circ}$. The response stimuli and task were the same as those used in Experiment 1

Design and procedure. The experimental design and procedure were identical to those of Experiment 1, with the exception that the directional cues were arrows and the nondirectional cue was a cross with perpendicular lines on the end of each arm. Cue-target SOA $(105,600,1,200$, or $1,800 \mathrm{~ms}$ ), cue type (left, right, up, or down arrow or nondirectional cross), and target identity ( $F$ or $T$ ) were selected randomly and with equal probability. The probabilities of a target appearing at any one of the four locations were the same as in Experiment 1. When the cue was an arrow pointing left, right, up, or down, the target letter appeared at the location opposite to where the arrow was pointing $75 \%$ of the time and at one of the other three locations $25 \%$ of the time; when the cue was the nondirectional cross, the target appeared with equal probability at any one of the four locations. Thus, there were four trial types, with probabilities identical to those in Experiment 1: (a) predicted trials, in which the target appeared at the predicted location (i.e., at the position opposite to where the arrow was pointing); (b) cued trials, in which the target appeared at the location toward which the arrow was pointing; (c) NP-NC trials, in which the target appeared at one of the two locations that were neither predicted nor pointed at by the arrow; and (d) nondirectional cross trials. Approximately $8 \%$ of the trials were catch trials, randomly selected from the five cue types.

As was the case with Experiment 1, each of the two experimental sessions was composed of 20 practice trials followed by 12 blocks of 60 trials, for a total of 1,440 test trials per subject. Figure 4 provides an illustration of the sequence of events on a test trial.

\section{Results}

Mean RTs, standard deviations, and error rates for Experiment 2 are presented in Table 2. As in Experiment 1, anticipations, timed-out trials, RTs longer than 1,000 ms, and incorrect responses

were classified as errors and were excluded from analysis. Each type of error accounted for less than $0.4 \%$ of the target trials. The false-alarm rate on catch trials was $1.38 \%$. Because these rates were so low, the error and false-alarm data were not analyzed further.

An ANOVA was conducted with SOA (105, 600, 1,200, and $1,800 \mathrm{~ms}$ ) and trial type (predicted, cued, and NP-NC) as withinsubject variables. Figure 5 illustrates RTs for predicted, cued, and NP-NC trials. As in Experiment 1, there was a significant main effect for SOA, $F(3,23)=27.89, M S E=635.06, p<.0001$, reflecting a foreperiod effect, and there was a significant main effect for trial type, $F(2,23)=36.55, M S E=232.73, p<.0001$, with RTs on predicted trials shorter overall than RTs on cued and NP-NC trials. The SOA $\times$ Trial Type interaction was also significant, $F(6,23)=5.23, M S E=192.41, p<.0001$.

Planned $t$ tests (Bonferroni corrected, two-tailed), conducted as in Experiment 1, revealed that performance in the predicted condition was significantly faster than in the NP-NC condition at all but the shortest SOA. This was precisely the same result that was observed in Experiment 1. However, unlike in Experiment 1, performance in the cued and NP-NC conditions was statistically equivalent at all SOAs. Recall that in Experiment 1, reflexive orienting was observed at the gazed-at location at both the 105and 600-ms SOAs.

An ANOVA with SOA $(105,600,1,200$, and 1,800 ms) and trial type (predicted, cued, and NP-NC) as within-subject variables and arrow type (symmetrical, asymmetrical) as a between-subjects variable revealed that there were no significant effects involving arrow type (all $F \mathrm{~s}<2$, $p \mathrm{~s}>.16$ ), confirming that arrow effects were equivalent when the arrow cues may have had less directional

Table 2

Mean Response Times (RTs; in Milliseconds), Standard Deviations, and Error Rates (\%E) for Experiment $2(N=24)$

\begin{tabular}{cccc}
$\begin{array}{c}\text { Cue-target SOA and } \\
\text { trial type }\end{array}$ & RT & SD & $\% \mathrm{E}$ \\
\hline 105 ms & & & \\
Predicted & 379 & 53 & 0.69 \\
Cued & 377 & 56 & 0.97 \\
NP-NC & 385 & 52 & 0.48 \\
Cross & 398 & 54 & 0.83 \\
600 ms & & & \\
Predicted & 348 & 52 & 0.78 \\
Cued & 374 & 61 & 0.19 \\
NP-NC & 372 & 50 & 0.76 \\
Cross & 366 & 46 & 0.44 \\
1,200 ms & & & 0.66 \\
Predicted & 333 & 49 & 0.74 \\
Cued & 355 & 50 & 0.56 \\
NP-NC & 353 & 45 & 0.56 \\
Cross & 349 & 46 & \\
1,800 ms & & & 0.81 \\
Predicted & 337 & 47 & 0.19 \\
Cued & 358 & 50 & 0.65 \\
NP-NC & 351 & 47 & 0.75 \\
Cross & 352 & 48 &
\end{tabular}

Note. Error rates represent the percentage of test trials from each cell excluded as anticipations, keypress selection errors, timed-out trials, or trials with $\mathrm{RT}>1,000 \mathrm{~ms}$. $\mathrm{SOA}=$ stimulus onset asynchrony; NP-NC $=$ not predicted-not cued. 


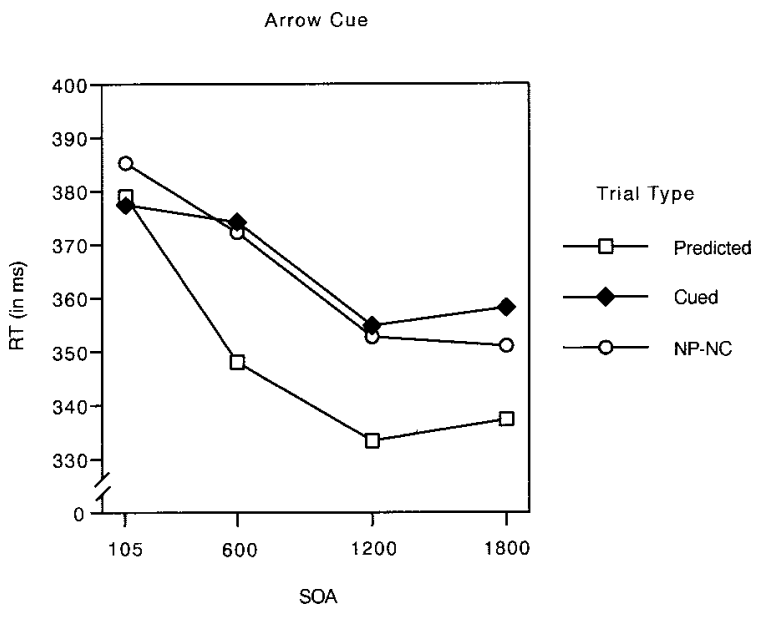

Figure 5. Mean response times (RTs) for counterpredictive arrow cues as a function of cue-target stimulus onset asynchrony (SOA) and trial type in Experiment 2. Predicted $=$ target occurs at the predicted (not-cued) location. Cued $=$ target occurs at the cued (not-predicted) location. NP-NC $=$ target occurs at a location that is neither predicted nor cued.

saliency than gaze cues (symmetrical arrows) and when the arrow cues were given greater directional weight (asymmetrical arrows).

As in Experiment 1, the 7 eye-monitored subjects rarely produced eye movements (3.0\%). A comparison of RT performance for these 7 eye-monitored subjects with that of the first 7 nonmonitored subjects produced no significant effects $\left(F_{\mathrm{s}}<1.4, p \mathrm{~s}>\right.$ $.20)$, once again confirming that eye movements were not involved in producing the cuing effects and that the orienting we observed was covert in nature.

\section{Discussion}

Experiment 2 was identical to Experiment 1, with the exception that arrows were used instead of gaze as the centrally presented counterpredictive cue. The pattern of RTs for predicted but notcued target locations versus locations that were neither predicted nor cued was very similar to that obtained with counterpredictive gaze in Experiment 1; that is, a reliable advantage for targets occurring at the predicted location was observed at the 600-, $1,200-$, and 1,800-ms SOAs, indicating that subjects were able to shift attention volitionally to the location where a target was likely to occur. However, the pattern of RTs for cued but not-predicted target locations versus locations that were neither predicted nor cued was very different from that obtained in Experiment 1: With arrows, there was never a significant advantage for targets occurring at the cued location. This difference between experiments was confirmed statistically, with an ANOVA comparing gaze and arrows (cue type) between groups and revealing significantly different cuing effects (trial type) both as a function of SOA-Cue Type $\times$ Trial Type $\times$ SOA: $F(9,46)=1.91, p<.05$ - and when collapsed across SOAs-Cue Type $\times$ Trial Type: $F(3,46)=7.87$, $p<.0001$. Note that this difference between gaze and arrows cannot be attributed to a perceptual weighting toward the cued (gazed-at) location in Experiment 1, because an asymmetrical arrow in Experiment 2 did not produce an advantage at the cued location, nor did it produce any difference from a symmetrical arrow.

Given the fact that nonpredictive arrows can produce relatively early facilitation (Ristic, Friesen, \& Kingstone, 2002), it may seem curious that in the present arrow-cuing experiment the advantage for cued target locations did not reach significance. This discrepancy may be due to differences in experimental design, such as the difference in the number of possible target locations (two in the Ristic et al., 2002, study and four in the present experiment), or differences in the distance between cued and uncued locations ( $180^{\circ}$ in the Ristic et al., 2002, study and $90^{\circ}$ in the present experiment). However, in recent experiments with nonpredictive arrows and four target locations (Ristic, Olk, Ho, \& Kingstone, 2003), we observed early facilitation similar to that observed in the Ristic et al. (2002) study with two target locations. Thus, we favor the more interesting and meaningful possibility that the discrepancy is due to differences in the predictive value of the arrow cues across studies. In the Ristic et al. (2002) study, the arrow cues were spatially nonpredictive. In the present experiment, they were spatially counterpredictive. It appears then that the arrow-cuing effect may be less strongly reflexive in nature than the gaze-cuing effect, and it may therefore be more vulnerable to subjects' top-down goals and expectations. If so, then it is reasonable that volitional orienting in a direction opposite to the arrow direction might undermine the reflexive orienting effect of the arrow stimuli in the present experiment. At any rate, our data indicate that, although gaze and arrows are similar in their ability to produce a volitional shift in covert attention, arrow cues do not trigger a reflexive shift of attention to a location where a target is unlikely to appear.

\section{General Discussion}

Our counterpredictive gaze experiment (Experiment 1) replicated the finding of Driver et al. (1999) that subjects orient attention reflexively to a gazed-at location at a short SOA even though they expect the target not to appear there. This confirms that orienting to gaze direction is reflexive in a strong sense, in that it can occur even against subjects' intentions. Experiment 1 also demonstrated that subjects can direct attention volitionally to a non-gazed-at location at longer SOAs. Moreover, the results of this experiment indicate that at an intermediate SOA, when both reflexive attention to the gazed-at location and voluntary attention to the likely location might be expected to occur, both may indeed have occurred. In other words, reflexive orienting to gazed-at locations and volitional orienting to likely locations exhibited different but overlapping time courses. This suggests that gazetriggered orienting and volitional orienting might occur somewhat independently of one another, such that attention can be directed reflexively to one location and volitionally to another location at the same time. Supplementary analyses supported this interpretation and failed to lend support to alternative explanations, such as the possibility that our finding of reflexive and volitional cooccurrence was an artifact of averaging across different subjects or the possibility that it was due to subjects switching between one type of orienting on one trial and another type of orienting on another trial.

The results of our second experiment, with counterpredictive arrows, suggest that the data pattern observed in Experiment 1 may be unique to gaze-direction cues. Experiment 2 was identical in 
every way to Experiment 1, with the exception that gaze-direction cues were substituted with arrow cues. Yet the results were clearly very different. In both experiments, evidence of covert voluntary orienting to the predicted target location was observed at 600-, $1,200-$, and 1,800-ms SOAs. However, in contrast to our findings with counterpredictive gaze cues, with counterpredictive arrow cues there was no evidence of covert reflexive orienting to the cued location. The difference observed in the present study between gaze and arrow cues lends support to the notion that gaze direction may be a special attentional cue that can trigger reflexive shifts of attention that are in opposition to, and concurrent with, volitional shifts of attention.

In the present study, we chose to use a schematic face, rather than an image of a real face, to provide the gaze cue because such a simple stimulus is more perceptually equivalent to other directional cues in the environment, such as the arrow cue we used. Nevertheless, one might wonder whether the gaze effects we observed with schematic faces can be generalized to more realistic looking faces and eyes. To our knowledge, only one study to date has directly compared the gaze-cuing effects of schematic faces with those of real faces. Using schematic and real faces with various emotional expressions, Hietanen and Leppänen (2003) found that schematic faces produced cuing effects similar to, albeit somewhat larger than, those produced by real faces at a $200-\mathrm{ms}$ SOA. In numerous other studies, the reflexive-gaze cuing effect has been observed with schematic faces (e.g., Friesen \& Kingstone, 1998, 2003a, 2003b; Kingstone, Friesen, \& Gazzaniga, 2000; Ristic, Friesen, \& Kingstone, 2002) and with real faces (e.g., Driver et al., 1999; Hietanen, 1999; Langton \& Bruce, 1999), and comparisons across studies suggest that, in general, the performance effects are equivalent. With regard more specifically to the effects of counterpredictive gaze cues, Experiment 1 of the present study, with schematic faces, replicated the findings produced by Driver et al. (1999, Experiment 3) with real faces (i.e., there is reflexive orienting to the gazed-at but unlikely target location at a short SOA, and there is no difference between cued and predicted locations at an intermediate SOA).

Our paradigm was designed so that we could assess the effects of the directional cues by comparing performance at cued and predicted locations with performance at locations that were neither predicted nor cued. The inclusion of trials on which the target would appear at a location that was neither predicted nor cued (NP-NC) by the directional stimulus (i.e., gaze or arrow) provided the ideal baseline for our purposes, because a target appearing at one of those locations was preceded by exactly the same type of cue as a target appearing at a cued location or a predicted location. As was first noted by Jonides and Mack (1984), failure to obtain such a baseline measure leaves open the very real possibility that performance differences between cued and neutral trials (e.g., the straight-gaze and cross cues, in the case of the present study) may have nothing to do with attentional orienting and everything to do with one or more confounding factors, such as arousal, effort, or strategy. Because we used baseline trials that were directional-cue trials, we can make assessments of reflexive and volitional orienting at different time windows with confidence-something that most studies have not been able to do (but see Kingstone \& Klein, 1991, and Danziger \& Kingstone, 1999, for two noteworthy exceptions).
In a previous study with nonpredictive gaze cues, in which straight-gaze trials were treated as a neutral baseline, Friesen and Kingstone (1998) concluded that gaze-direction cues produced benefits at gazed-at locations without any corresponding costs at non-gazed-at locations. The inclusion of similar neutral trials (straight-gaze trials in Experiment 1 and cross trials in Experiment 2 ) in the design of the present study afforded us an opportunity to compare the neutral nondirectional-cue trials (straight gaze or cross) with our NP-NC directional-cue trials. For each experiment, an ANOVA was conducted with trial type (directional NP-NC, nondirectional neutral) and SOA $(105,600,1,200,1,800 \mathrm{~ms})$ as within-subject variables. For gaze cues (Experiment 1), there was a main effect for trial type, with RT 5 ms longer on NP-NC trials than on straight-gaze trials, $F(1,23)=11.11, p<.005$, and the Trial Type $\times$ SOA interaction was not significant $(F<1.0)$. For arrow cues (Experiment 2), the main effect for trial type was not significant $(F<1.0)$, but the SOA $\times$ Trial Type interaction was significant, $F(3,23)=7.11, p<.0005$. Inspection of the data suggested that this interaction was caused by shorter RTs on NP-NC trials than on central-cross trials at the 105-ms SOA. In agreement with this interpretation, when the 105-ms SOA trials were removed from the ANOVA, a completely different result was obtained. Now, there was a marginally significant main effect for cue type, with RT 3 ms longer on NP-NC trials than on cross trials, $F(1,23)=3.11, p<.10$, and the Trial Type $\times$ SOA interaction fell far short of significance $(F<1.6)$.

The overall pattern of results with the nondirectional trials converges with the results we reported using directional trials as our baseline. Both indicate that the RT advantage for both cued locations and predicted locations represents facilitation. As for the one anomalous finding just discussed (shorter RT on NP-NC arrow trials than on central-cross trials at the 105-ms SOA), it provides an illustration of the inherent danger of failing to include an appropriate baseline measure at the time that attention is cued (Jonides \& Mack, 1984). If we had not included directional baseline NP-NC trials in our experiment, and if we had compared predicted and cued trials with the nondirectional-cross trials, we would have been misled into thinking that there was early facilitation on both predicted trials and cued trials. But the data reveal that directional baseline trials are also "facilitated" relative to neutral trials. Clearly, there really was no cuing effect occurring at the 105-ms SOA (predicted and cued trials were not significantly faster than NP-NC trials), and the neutral nondirectional stimulus (i.e., the cross) was treated differently from the directional arrow cues at this early SOA. Thus, it is important to note that although nondirectional neutral cues (e.g., our straight-gaze and cross cues) might generally serve as a reasonable baseline, they do not always do so.

The different but overlapping time courses of reflexive orienting to a gazed-at location and volitional orienting to a likely target location observed in Experiment 1 suggest that the two forms of orienting may be independent and, thus, that they may be subserved by different attentional systems or subsystems. There is considerable evidence in the attentional literature indicating that reflexive orienting to a sudden onset at a peripheral location and volitional orienting to an expected target location occur by way of different brain pathways. Reflexive orienting to a sudden onset in the periphery is thought to involve the superior colliculus (SC), working in concert with parietal cortex (Rafal, Henik, \& Smith, 
1991; Rafal, Posner, Friedman, Inhoff, \& Bernstein, 1988), whereas volitional orienting to an expected target location is thought to involve frontal and parietal areas (Corbetta, Miezin, Shulman, \& Petersen, 1993; Posner, 1995; Posner \& Raichle, 1994). It seems likely, however, that reflexive orienting triggered by gaze direction does not occur by way of either of these pathways.

Several lines of evidence suggest that gaze-triggered orienting does not occur by way of the subcortical pathway. First, in their study with split-brain patients, Kingstone, Friesen, and Gazzaniga (2000) demonstrated that reflexive orienting to gaze direction is lateralized to one cortical hemisphere. Second, in a recent eyemovement study, Friesen and Kingstone (2003b) found that gazedirection cues did not activate or predisengage the oculomotor system, suggesting that orienting to gaze direction does not engage the SC. Third, Friesen and Kingstone (2003a) demonstrated that reflexive orienting to gaze direction can co-occur with IOR (which is subserved by the SC).

In a similar manner, the finding of Experiment 1 of the present study that reflexive orienting to a gazed-at location and volitional orienting to a different location might co-occur suggests that attention to gaze does not occur by way of the frontal-parietal pathway that underlies volitional orienting. This conclusion is consistent with three other results suggesting that gaze-triggered orienting is not simply a well-learned form of volitional orienting. First, Ristic, Friesen, and Kingstone (2002) found that preschool children showed greater orienting effects than adults in response to nonpredictive gaze-direction cues, despite the fact that young children are thought to be poor at volitional orienting (Brodeur, Trick, \& Enns, 1997). Second, Hood, Willen, and Driver (1998) found that infants were faster to make saccades to peripheral targets that were cued nonpredictively by the gaze direction of a central face and concluded that gaze-triggered orienting is in place very early in development (but see Farroni, Johnson, Brockbank, \& Simion, 2000, for an alternative explanation). Third, in their split-brain patient study, Kingstone et al. (2000) found that although only the cortical hemisphere specialized for face and gaze processing oriented reflexively in response to nonpredictive gaze cues, both hemispheres oriented volitionally in response to predictive gaze cues.

So, what might the gaze-triggered reflexive attention pathway be? Kingstone et al. (2000) proposed that orienting to gaze direction might be subserved by a temporal-parietal pathway, with cells in inferotemporal cortex processing face and eye information, cells in the superior temporal sulcus processing the direction of gaze, and cells in parietal cortex shifting attention to the gazed-at location. Each of these brain regions has since been implicated in gaze-direction processing in a number of human neuroimaging studies (e.g., Hoffman \& Haxby, 2000; Kato et al., 2001; Puce, Allison, Bentin, Gore, \& McCarthy, 1998; Wicker, Michel, Henaff, \& Decety, 1998). Note that all three of the attentional pathways discussed here-the subcortical reflexive pathway, the cortical volitional pathway, and the proposed cortical gazedirection pathway-involve parietal cortex. How, then, could attention be shifted reflexively to a gazed-at location and volitionally to a different location at the same time? One possibility is that volitional inputs from frontal cortex and gaze inputs from temporal cortex activate different parietal neurons. In a recent functional MRI study that compared peripheral target detection and volitional orienting, Corbetta, Kincade, Ollinger, McAvoy, and Schulman (2000) found evidence for this type of dissociation, with temporoparietal cortex activated during target detection and intraparietal cortex activated during volitional orienting.

To our knowledge, the present study is the first to demonstrate that gaze cues and arrow cues can produce qualitatively different behavioral results in intact subjects. In their recent study with nonpredictive gaze and arrow cues, Ristic et al. (2002) found that nonpredictive gaze cues and nonpredictive arrow cues produced similar RT patterns in normal subjects (both adults and children). Differences in the effects of the two types of directional cue were revealed only when the performance of a split-brain patient was examined: Nonpredictive arrow cues triggered orienting in both hemispheres, whereas in a previous study of the same patient (Kingstone et al., 2000), nonpredictive gaze cues triggered orienting only in the hemisphere specialized for face processing. On the basis of this difference in lateralization for the two cue types, Ristic et al. concluded that gaze is indeed special. The present study, however, demonstrates that apart from the issue of lateralization of face processing, gaze and arrow cues can trigger qualitatively different behavioral effects. When each of these directional cues is put into competition with volitional orienting, orienting to gaze direction persists, whereas orienting to arrows is abolished.

In sum, the results of the present study confirm that attentional orienting toward a gazed-at location is reflexive, not only in the sense that it occurs when subjects do not have any incentive to attend to the gazed-at location (as is the case in nonpredictive-gaze experiments) but also in the stronger sense that it can occur even when subjects are attending volitionally to an opposite location. Our finding that reflexive and volitional orienting in response to gaze direction appear to co-occur suggests that the two may be subserved by distinct and separable mechanisms. Arrow cues can also produce reflexive shifts of attention (Ristic et al., 2002; Tipples, 2002), but unlike eyes, they do not do so when they are counterpredictive. The implication is that although many directional cues might trigger reflexive shifts of attention when they are spatially nonpredictive, they are not all equal. In particular, gaze cues appear to be more strongly reflexive than arrow cues, very possibly because they access a neural architecture that is specialized for processing eye direction.

\section{References}

Brodeur, A. D., Trick, M. I., \& Enns, J. T. (1997). Selective attention over the lifespan. In A. J. Burack \& J. T. Enns (Eds.), Attention, development, and psychopathology (pp. 74-97). New York: Guilford Press.

Cheal, M. L., \& Lyon, D. R. (1991). Central and peripheral precuing of forced-choice discrimination. Quarterly Journal of Experimental Psychology: Human Experimental Psychology, 43(A), 859-880.

Corbetta, M., Kincade, J. M., Ollinger, J. M., McAvoy, M. P., \& Schulman, G. L. (2000). Voluntary orienting is dissociated from target detection in human posterior parietal cortex. Nature Neuroscience, 3, 292-297.

Corbetta, M., Miezin, F. M., Shulman, G. L., \& Petersen, S. E. (1993). A PET study of visuospatial attention. Journal of Neuroscience, 13, 12021226.

Danziger, S., \& Kingstone, A. (1999). Unmasking the inhibition of return phenomenon. Perception \& Psychophysics, 61, 1024-1037.

Driver, J., Davis, G., Ricciardelli, P., Kidd, P., Maxwell, E., \& BaronCohen, S. (1999). Gaze perception triggers visuospatial orienting by adults in a reflexive manner. Visual Cognition, 6, 509-540. 
Farroni, T., Johnson, M. H., Brockbank, M., \& Simion, F. (2000). Infants' use of gaze direction to cue attention: The importance of perceived motion. Visual Cognition, 7, 705-718.

Friesen, C. K., \& Kingstone, A. (1998). The eyes have it! Reflexive orienting is triggered by nonpredictive gaze. Psychonomic Bulletin \& Review, 5, 490-495.

Friesen, C. K., \& Kingstone, A. (2003a). Abrupt onsets and gaze direction cues trigger independent reflexive attentional effects. Cognition, 87, B1-B10.

Friesen, C. K., \& Kingstone, A. (2003b). Covert and overt orienting to gaze direction cues and the effects of fixation offset. NeuroReport, 14, 489493.

Hietanen, J. K. (1999). Does your gaze direction and head orientation shift my visual attention? NeuroReport, 10, 3443-3447.

Hietanen, J. K., \& Leppänen, J. M. (2003). Does facial expression affect attention orienting by gaze direction cues? Journal of Experimental Psychology: Human Perception and Performance, 29, 1228-1243.

Hoffman, E. A., \& Haxby, J. V. (2000). Distinct representations of eye gaze and identity in the distributed human neural system for face perception. Nature Neuroscience, 3, 80-84.

Hood, B. M., Willen, J. D., \& Driver, J. (1998). Adults' eyes trigger shifts of visual attention in human infants. Psychological Science, 9, 131-134.

Jonides, J. (1981). Voluntary versus automatic control over the mind's eye's movement. In J. B. Long \& A. D. Baddeley (Eds.), Attention and performance IX (pp. 187-203). Hillsdale, NJ: Erlbaum.

Jonides, J., \& Mack, R. (1984). On the cost and benefit of cost and benefit. Psychological Bulletin, 96, 29-44.

Kato, C., Matsuo, K., Matsuzawa, M., Moriya, T., Glover, G. H., \& Nakai, T. (2001). Activation during endogenous orienting of visual attention using symbolic pointers in the human parietal and frontal cortices: A functional magnetic resonance imaging study. Neuroscience Letters, $314,5-8$.

Kingstone, A., Friesen, C. K., \& Gazzaniga, M. S. (2000). Reflexive joint attention depends on lateralized cortical connections. Psychological Science, 11, 159-165.

Kingstone, A., \& Klein, R. (1991). Combining shape and position expectancies: Hierarchical processing and selective inhibition. Journal of Experimental Psychology: Human Perception and Performance, 17, 512519

Kingstone, A., \& Pratt, J. (1999). Inhibition of return is composed of attentional and oculomotor processes. Perception \& Psychophysics, 61, $1046-1054$

Kingstone, A., Smilek, D., Ristic, J., Friesen, C. K., \& Eastwood, J. D. (2003). Attention, researchers! It is time to take a look at the real world. Current Directions in Psychological Science, 12, 176-180.

Klein, R. M. (2000). Inhibition of return. Trends in Cognitive Sciences, 4, $138-147$.

Klein, R. M., Kingstone, A., \& Pontefract, A. (1992). Orienting of visual attention. In K. Rayner (Ed.), Eye movements and visual cognition: Scene perception and reading (pp. 46-63). Amsterdam: Elsevier Science.

Langton, S. R. H., \& Bruce, V. (1999). Reflexive social orienting. Visual Cognition, 6, 541-567.
Langton, S. R. H., Watt, R. J., \& Bruce, V. (2000). Do the eyes have it? Cues to the direction of social attention. Trends in Cognitive Sciences, 4, $50-59$.

Mowrer, O. H. (1940). Preparatory set (expectancy)—Some methods of measurements. Psychological Review Monograph, 52(Whole No. 233).

Müller, H. J., \& Rabbitt, P. M. A. (1989). Reflexive and voluntary orienting of visual attention: Time course of activation and resistance to interruption. Journal of Experimental Psychology: Human Perception and Performance, 15, 315-330.

Posner, M. I. (1980). Orienting of attention. Quarterly Journal of Experimental Psychology, 32, 3-25.

Posner, M. I. (1995). Attention in cognitive neuroscience: An overview. In M. S. Gazzaniga (Ed.), The cognitive neurosciences (pp. 615-624). Cambridge, MA: MIT Press.

Posner, M. I., \& Cohen, Y. (1984). Components of visual orienting. In H. Bouma \& D. G. Bouwhuis (Eds.), Attention and performance X: Control of language processes (pp. 531-556). Hillsdale, NJ: Erlbaum.

Posner, M. I., Cohen, Y., \& Rafal, R. D. (1982). Neural systems control of spatial orienting. Philosophical Transactions of the Royal Society of London, Series B, 298, 187-198.

Posner, M. I., Rafal, R. D., Choate, L. S., \& Vaughan, J. (1985). Inhibition of return: Neural basis and function. Cognitive Neuropsychology, 2, 211-228.

Posner, M. I., \& Raichle, M. E. (1994). Images of mind. New York: Scientific American Library.

Puce, A., Allison, T., Bentin, S., Gore, J. C., \& McCarthy, G. (1998). Temporal cortex activation in humans viewing eye and mouth movements. Journal of Neuroscience, 18, 2188-2199.

Rafal, R., Henik, A., \& Smith, J. (1991). Extrageniculate contributions to reflex visual orienting in normal humans: A temporal hemifield advantage. Journal of Cognitive Neuroscience, 3, 322-328.

Rafal, R. D., Posner, M. I., Friedman, J. F., Inhoff, A. W., \& Bernstein, E. (1988). Orienting of visual attention in progressive supranuclear palsy. Brain, 111, 267-280.

Rensink, R. A. (1995). VScope (Version 1.2.7) [Computer software]. Vancouver, British Columbia, Canada: Micropsych Software.

Ristic, J., Friesen, C. K., \& Kingstone, A. (2002). Are eyes special? It depends on how you look at it. Psychonomic Bulletin \& Review, 9, 507-513.

Ristic, J., Olk, B., Ho, S., \& Kingstone, A. (2003, April). Endogenous orienting: What have we been measuring? Poster presented at the 10th Annual Meeting of the Cognitive Neuroscience Society, New York.

Tipples, J. (2002). Eye gaze is not unique: Automatic orienting in response to uninformative arrows. Psychonomic Bulletin \& Review, 9, 314-318.

Wicker, B. F., Michel, F., Henaff, M., \& Decety, J. (1998). Brain regions involved in the perception of gaze: A PET study. Neuroimage, 8, 221-227.

Received October 30, 2002

Revision received September 29, 2003

Accepted September 29, 2003 